\title{
Balkanologie
}

Revue d'études pluridisciplinaires

Vol. $15 n^{\circ} 2 \mid 2020$

Pour une approche socio-historique de l'action collective dans les Balkans

\section{Entre le local, le national et le global. Appréhender les mouvements sociaux en Europe du Sud-Est}

Florian Bieber, Dario Brentin (dir.), Social Movements in the Balkans. Rebellion and Protest from Maribor to Taksim, Londres, Routledge, 2018 | Adam Fagan, Indraneel Sircar (dir.), Activist Citizenship in Southeast Europe, Londres, Routledge, 2018

Fabio Giomi et Julia Nietsch

\section{(2) OpenEdition}

1 Journals

Édition électronique

URL : http://journals.openedition.org/balkanologie/2644

DOI : 10.4000/balkanologie.2644

ISSN : 1965-0582

Éditeur

Association française d'études sur les Balkans (Afebalk)

Référence électronique

Fabio Giomi et Julia Nietsch, «Entre le local, le national et le global. Appréhender les mouvements sociaux en Europe du Sud-Est », Balkanologie [En ligne], Vol. 15 n $^{\circ}$ | 2020, mis en ligne le 01

décembre 2020, consulté le 23 avril 2021. URL : http://journals.openedition.org/balkanologie/2644 ; DOI : https://doi.org/10.4000/balkanologie.2644

Ce document a été généré automatiquement le 23 avril 2021.

(c) Tous droits réservés 


\section{Entre le local, le national et le global. Appréhender les mouvements sociaux en Europe du Sud-Est}

Florian Bieber, Dario Brentin (dir.), Social Movements in the Balkans. Rebellion and Protest from Maribor to Taksim, Londres, Routledge, 2018 | Adam Fagan, Indraneel Sircar (dir.), Activist Citizenship in Southeast Europe, Londres, Routledge, 2018

Fabio Giomi et Julia Nietsch

\section{RÉFÉRENCE}

Bieber Florian, Brentin Dario (dir.), Social Movements in the Balkans. Rebellion and Protest from Maribor to Taksim, Londres, Routledge, 2018, 200 pages, ISBN 978-1-138-05214-7 Fagan Adam, Sircar Indraneel (dir.), Activist Citizenship in Southeast Europe, Londres, Routledge, 2018, 176 pages, ISBN 978-1138604896

Ces dernières années, les mouvements sociaux ont éveillé l'intérêt des praticiens des sciences sociales travaillant sur l'Europe du Sud-Est. Ce nouveau corpus de recherches, qui relève à la fois de la science politique, de la sociologie, de l'anthropologie, mais aussi de la géographie et des études de genre, met en cause l'un des lieux communs les plus coriaces: celui selon lequel les sociétés civiles de cette partie du continent européen seraient fatalement faibles, défectueuses, voire absentes. Selon ce discours, dont le succès dépasse largement le périmètre des publications scientifiques, la cause de cette défaillance se trouverait dans l'histoire récente, notamment dans l'expérience socialiste et postsocialiste ${ }^{1}$; ce qui ne fait qu'alimenter un discours savant plus ancien que Maria Todorova a qualifié de «balkaniste " et qui assigne à la longue durée, 
notamment à l'intégration de la région dans l'Empire ottoman pendant plusieurs siècles, la cause de cette faiblesse chronique ${ }^{2}$.

2 Ce compte rendu se propose d'opérer une lecture croisée de deux volumes collectifs publiés en 2018, Social Movements in the Balkans. Rebellion and Protest from Maribor to Taksim, dirigé par Florian Bieber et Dario Brentin, et Activist Citizenship in Southeast Europe, dirigé par Adam Fagan et Indraneel Sircar ${ }^{3}$. Au-delà de leur structure (deux livres dirigés, d'une dizaine de contributions chacun), les deux textes ont d'autres traits en commun qui les rendent facilement comparables, notamment leur matrice disciplinaire. En effet, les quatre directeurs d'ouvrages relèvent tous des différentes branches de la science politique. Si Florian Bieber et Dario Brentin, au moins au moment de la publication du livre, sont affiliés à l'Université de Graz, plus précisément au Centre for Southeast European Studies (CSEES), Adam Fagan et Indraneel Sircar sont respectivement rattachées à l'Université Queen Mary et à la London School of Economics and Political Science. Il s'agit de chercheurs avec une expertise aréale reconnue, qui ont déjà publié sur l'Europe du Sud-Est, et plus généralement sur l'Europe orientale.

3 Les deux volumes proposent une lecture double de l'action collective des années 2010 en Europe du Sud-Est: une lecture diachronique, ancrée dans l'histoire des mouvements sociaux de différentes villes ou pays, et une autre synchronique, attentive aux échanges avec d'autres mouvements sociaux en Europe et au Moyen-Orient ${ }^{4}$. Ainsi, les auteur.e.s portent une attention particulière aux transformations des décennies précédentes, caractérisées - à l'exception de la Grèce - par l'effondrement des régimes socialistes et par le passage à des systèmes économiques de libre marché - passage qui, dans l'espace yougoslave, a été accompagné par des guerres de dissolution particulièrement destructrices (1991-1999).

Les deux volumes pointent du doigt les mêmes spécificités par rapport aux mouvements sociaux des années 2010 : il s'agirait d'un engagement qui, même s'il est souvent déclenché par des épisodes très situés (la destruction d'un parc, la fermeture d'un hôpital, etc.), sert rapidement à exprimer « un mécontentement assez large, en même temps politique, social et économique $»^{5}$ vis-à-vis des politiques d'austérité sévères mises en place suite à la crise financière. Tant dans le cas où la protestation monte explicitement en généralité que dans les cas où elle reste " discrète " ${ }^{6}$, ce sont les élites du postsocialisme qui sont au centre de la critique, en particulier les élites gouvernementales, économique et partisanes, accusées d'avoir trahi les promesses de la «transition » à la démocratie et au libre marché. Ces élites nationales n'auraient pas su ou pas voulu protéger les communs, c'est à dire les espaces, biens et services publics, une inaction qui amène Bieber et Brentin à parler, en faisant référence au texte désormais classique de Garret Hardin ${ }^{7}$, d'une "nouvelle tragédie des communs ${ }^{8}$ en Europe du Sud-Est. Mais la critique ne s'arrête pas à l'échelle nationale, s'adressant aussi aux acteurs publics et privés supranationaux: les institutions de l'Union Européenne in primis, accusées d'être le moteur des privatisations des dernières décennies qui ont finalement profité aux réseaux clientélistes des partis au pouvoir, ainsi que le secteur des ONG qui, depuis les années 1990, est devenu un acteur structurant des sociétés civiles de l'Europe du Sud-Est. Il s'agit en somme, pour utiliser la classification que Paul Stubbs a élaborée à partir du cas croate ${ }^{9}$, des mouvements de la « troisième vague » : la première vague étant constituée par les mouvements pour la démocratie et (dans l'espace yougoslave) contre la guerre dans les années 1980-1990; la 
deuxième vague étant celle des ONG professionnalisées et dépendantes des bailleurs internationaux des années 1990 et 2000 ; la troisième étant, enfin, une réaction à la bureaucratisation et - pour utiliser l'expression de Jacobson and Saxonberg - à l'ONGisation ${ }^{10}$, aux politiques d'austérité et pour le droit à la ville. Au prix d'un certain degré de simplification, il s'agit de mouvements que l'on peut considérer comme relevant du périmètre de la gauche, les mouvements sociaux « de droite » n'étant pas analysés dans ces deux ouvrages.

5 Un autre trait qui rapproche les deux volumes est l'ambition multiscalaire. Les introductions comme les différents chapitres s'efforcent d'appréhender les mouvements sociaux à des échelles d'analyse différentes : locale (un site en particulier, une ville, un village), étatique (arène privilégiée pour la politique nationale), régionale (labellisée «Balkans» dans le premier volume, «Europe du Sud-Est» dans le deuxième), mais aussi globale. Sur l'importance de cette dernière échelle d'analyse, Bieber et Brentin sont clairs: "hétérogènes dans leurs nature et impact, ces mouvements de protestation passés et présents doivent être compris comme faisant partie d'une plus large vague de mouvements sociaux qui se déroulent à l'échelle globale : de Occupy Wall Street aux États-Unis à Maidan en Ukraine, de Gezi Park en Turquie à la Place Tahrir en Égypte ${ }^{11} »$. Pour dépasser une logique purement nationale des cas d'étude, qui néanmoins organise la plupart des contributions, plusieurs articles du même volume font l'effort d'aborder la région dans son ensemble, ou bien un groupe de pays dans une perspective comparée.

6 La capacité des deux ouvrages à couvrir l'aire qu'ils évoquent dans le titre est toutefois différente : si le volume de Bieber et Brentin, comme annoncé dans le sous-titre « from Maribor to Taksim », a une focale plus large et englobe des cas d'études venant de Turquie, Bosnie-Herzégovine, Slovénie, Bulgarie, Grèce, le texte de Fagan et Sircar est essentiellement post-yougoslave (Bosnie-Herzégovine, Serbie, Slovénie, Croatie, Vojvodine), avec l'ajout d'un article sur la Roumanie.

7 Les deux volumes diffèrent non seulement dans leur périmètre géographique, mais aussi et surtout dans leur parti-pris théorique et méthodologique. Ainsi que l'indique le choix de leur titre, Bieber et Brentin situent leur ouvrage collectif dans la tradition des Social Movement Studies et de l'étude des politiques du conflit (contentious politics) ${ }^{12}$, dans une perspective explicitement comparative et transnationale. Les études de cas présentées dans le volume interrogent la manière dont les mouvements sociaux confrontent les représentants de l'État et, plus généralement, la crise de légitimité de la démocratie représentative. Comme le montre par exemple Valentina Gueorguieva dans sa contribution sur le «Printemps bulgare» de 2012-2013, les acteurs de ces mouvements ne luttent pas contre, mais pour l'État, vers lequel ils se tournent pour demander une meilleure protection des biens communs. Ceci constitue d'ailleurs, selon l'auteure, un paradoxe, puisque les manifestants sont bien conscients de la corruption et de l'inefficacité des institutions - un activiste-écrivain cité par l'auteure va même jusqu'à déplorer un "État détruit » (ruined) qui "n'est même pas un État " $^{13}$. Ce cadre analytique, qui établit une relation étroite entre mouvement social et État, amène à percevoir les activistes essentiellement comme "des freins (checks) à l'action des dirigeants autoritaires, corrompus ou irresponsables $»^{14}$, comme l'écrit Heiko Wimmen dans son texte sur les collectifs citoyens tels que Dosta ou GROZD en BosnieHerzégovine. Cette perspective d'analyse centrée sur un État « capturé $~^{15}$ par les élites postsocialistes n'est pas sans rappeler le "paradigme de transition démocratique et 
économique » que Bieber et Brentin disent pourtant critiquer, mais qui transparaît tout de même (involontairement?) à certains endroits de l'ouvrage, par exemple quand Dosta et GROZD sont décrits par Wimmen comme une "étape » vers une société pluraliste, plus démocratique et tolérante.

8 Plutôt que de privilégier une approche nationale ou internationale et de se concentrer sur la relation avec le pouvoir, Fagan et Sircar cherchent à démontrer l'originalité des engagements (ultra-)locaux, afin d'invalider les hypothèses de l'«apathie » postsocialiste et de la «faiblesse » des sociétés civiles de cette région, à laquelle on assigne un statut périphérique et retardataire par rapport à l'Europe occidentale. Dans leur ouvrage, les contributions ne traitent pas de mouvements sociaux, mais insistent, comme l'annonce le titre, sur le lien entre activisme et citoyenneté, en s'inspirant de la notion d' «activist citizenship». Selon Engin Fahri Isin, chercheur auquel on doit ce concept, le citoyen activiste formule des requêtes, brise l'habitus, trouble l'ordre, les pratiques et les statuts établis ${ }^{16}$. Le prisme de l'activisme citoyen amène les contributeur.trice.s à analyser de plus près des engagements à petite échelle qui ne sont pas forcément visibles et mesurables. Ainsi, Piotr Goldstein étudie l'engagement des libraires de Novi Sad qui, par le choix des livres qu'ils proposent et par les événements qu'ils organisent, constituent des "contre-espaces ${ }^{17}$, des espaces d'engagement sociétal et de co-création avec leur public. Pour Goldstein, l'engagement quotidien de ces libraires et des activistes qui profitent de leurs librairies pour y organiser des activités contribue à créer des "réalités alternatives ${ }^{18}$. Ainsi, ce deuxième volume vise à mettre en lumière les conditions locales de l'émergence des protestations et les différentes pratiques de mobilisation des ressources.

9 En parcourant les deux volumes, on est avant tout frappés par l'extraordinaire variété des types d'actions considérées légitimes par les acteurs pour se faire entendre sur une problématique donnée - ce que Charles Tilly a appelé en 1984 le répertoire d'action collective $^{19}$. Ces répertoires d'action puisent dans une pluralité de capitaux culturels, venant autant de leur propre passé que des activités d'autres mouvements sociaux des années 2010 dans le monde. Afin de rendre justice à cette diversité, les textes des deux ouvrages se nourrissent de différentes disciplines (science politique, histoire, sociologie, anthropologie, études visuelles, études de genre) et méthodologies (quantitatives ou qualitatives). Le texte de Ksenija Berk, qui porte sur la culture visuelle de la protestation en Slovénie, montre par exemple comment cette dernière mobilise et mélange des références provenant du mouvement de libération national slovène pendant la Grande Guerre, des avant-gardes artistiques socialistes yougoslaves, ainsi que des références à des films indépendants américains et à la culture populaire, comme les zombies, ou encore des références aux mouvements de contestation internationaux, comme le montre le recours aux masques de Guy Fawkes ${ }^{20}$. Les circulations transnationales des personnes, des discours et des pratiques entre mouvements sont aussi au centre du chapitre d'Astrid Reinprecht, qui analyse les transferts entre les mouvements étudiants en France, au Royaume-Uni, en Grèce et les assemblées d'étudiants en Serbie en 2006, qui serviront à leur tour de modèle pour les étudiants de Zagreb ${ }^{21}$.

10 Les contributions des deux volumes montrent également combien ces répertoires d'action collective peuvent changer, même dans un temps relativement réduit, et donc combien les stratégies de ces mouvements sont tout sauf figées. C'est par exemple ce que montre Chiara Milan, qui insiste beaucoup sur la capacité des activistes bosniens 
engagés dans la Bebolucija en 2013 puis dans les Plenums en 2014 à avoir recours à des outils différents, allant des rassemblements, sit-in, manifestations et blocages de sites à des confrontations plus violentes ${ }^{22}$. Souvent, ce changement est rendu nécessaire par un changement de l'échelle de la mobilisation, en particulier quand il y a une montée en puissance rapide. C'est le cas des mobilisations de 2012 en Slovénie, analysées par Gal Kirn, qui sont déclenchées contre la municipalité de Maribor et qui deviennent rapidement un phénomène national, qui vise l'élite politique toute entière. Pour Kirn, cette "irruption démocratique massive" de ceux qui sont "invisibles» et qui reprennent contrôle de la politique ne peut être totalement expliquée par des facteurs extérieurs, tels que les conditions économiques, mais renvoie à l'« intériorité » de la politique $^{23}$. Une transformation similaire des répertoires est détectée par Alen Toplišek et Lasse Thomassen dans leur analyse de la transformation du mouvement de la gauche slovène en parti politique ${ }^{24}$.

11 L'attention portée aux changements de répertoires représente probablement l'apport le plus enrichissant de ces deux volumes, ainsi que l'analyse de la circulation des discours et pratiques d'activisme dans le «cycle global de protestation ${ }^{25}$ " post-2008, pour utiliser l'expression de Chiara Milan et de Leonidas Oikonomakis. Néanmoins, il faut signaler un bémol, notamment dans le volume de Bieber et Brentin: les circulations internationales, les contacts et les émulations entre mouvements sont omniprésents, mais rarement explorés en profondeur. Comment et par qui s'établit le lien entre les activistes de l'Europe du Sud-Est et les activistes d'autres parties de l'Europe, des États-Unis ou du Moyen-Orient? Est-ce que les activistes des différents pays s'inspirent les uns les autres par le biais des médias? Ou est-ce qu'ils/elles se rencontrent et échangent de manière plus personnelle et structurée ? Est-ce que les circulations vont dans un seul sens ou dans les deux? En lisant, on aurait voulu en savoir plus sur la matérialité de ces circulations, sur les circonstances et les canaux qui les rendent possibles.

12 Les deux collections d'articles montrent bien comment les activistes font émerger un mouvement social en jonglant entre les espaces physiques et virtuels. Pour Danijela Dolenec, Karin Doolan et Tomislav Tomašević qui étudient le mouvement pour le droit à la ville de Zagreb, les mouvements sociaux sont de véritables artisans de la ville, capables de formuler une critique radicale des transformations postsocialiste et néolibérale ${ }^{26}$. Kostas Plevris analyse la relation entre mobilisations et espace urbain en Grèce depuis 2008 et montre de façon convaincante que les mouvements sociaux proposent non seulement une transformation de la vie quotidienne, mais aussi une nouvelle organisation de l'espace social ${ }^{27}$. Sans surprise, pratiquement tous les articles soulignent la familiarité des activistes avec Internet et avec les réseaux sociaux, qui leur permettent de s'auto-organiser et de créer un espace contre-discursif par rapport aux médias grand public, souvent très proches du gouvernement. Comme le dit en particulier Željka Lekić-Subašić, dans les différents mouvements sociaux qui ont vu le jour en Europe du Sud-Est, les activités en ligne «ont rendu la communication plus fréquente et intense, et elles ont créé des sentiments d'empathie et d'appartenance au même groupe "; à noter aussi que les activités en ligne "ne se substituent pas aux actions politiques que les citoyens engagés mènent dans la vie réelle, mais en constituent un volet ${ }^{28} »$.

13 Un dernier point concerne la sociologie de ces mouvements. L'étude de Marius I. Tatar, qui compare, à partir d'une analyse quantitative, la disposition à signer des pétitions et 
à descendre dans la rue dans les différents pays de la région, nous aide à comprendre qui sont les personnes qui animent les mouvements de ces dernières décennies ainsi que leurs spécificités sociologiques ${ }^{29}$. La plupart des contributions insistent sur l'importance de la présence d'artistes dans les mobilisations et sur leurs apports en termes de communication intégrant mots, images et actions. Les performances artistiques jouent un rôle majeur, par exemple, lors des manifestations du «Printemps bulgare " dans les villes principales du pays, retracées par Gueorguieva à travers les souvenirs des hommes et des femmes qui y ont participé. Cette implication des artistes, qui « attire l'attention des médias et des passants ${ }^{30}$ ", n'est d'ailleurs pas une exclusivité des espaces urbains. Comme le montre bien Bojan Baća, les habitants du village de Beranselo, près de Berane au Monténégro, qui protestent, entre 2007 et 2014, contre la construction d'une décharge de déchets, arrivent à impliquer des artistes dans leur mouvement et à utiliser des stratégies de communication inspirées des arts visuels ${ }^{31}$. Nous aurions souhaité que les auteur.e.s des textes des deux recueils portent une attention plus systématique à la variable du genre qui, quand elle est mobilisée, montre tout son potentiel heuristique, comme dans l'article d'Alexandra Ana qui éclaire la manière dont les mouvements féministes « influencent et contestent la dynamique des mobilisations ${ }^{32}$ » de 2012 en Roumanie.

Pour conclure, ces deux volumes représentent un acquis important pour notre compréhension des mouvements sociaux en Europe du Sud-Est, de l'articulation entre les échelles micro et macro, des connections transnationales et des spécificités par rapport aux vagues précédentes. En effet, l'un des constats de ces deux ouvrages est que les mobilisations des années 2010 ont une spécificité par rapport aux décennies précédentes, mais qu'il n'y a pas forcément une spécificité aréale. Plus généralement, ces recueils contribuent à une perception plus nuancée et complexe des sociétés de la région, souvent imaginées comme étant en retard, passives et - au moins en ce qui concerne l'espace post-yougoslave - éternellement dominées par l'ethnicité et les nationalismes. Les deux ouvrages montrent au public anglophone que l'Europe du SudEst est pleine de vie et de créativité et, surtout, que cette partie de l'Europe est inextricablement connectée au « reste » du monde.

\section{NOTES}

1. À ce propos, voir par exemple la thèse de HOWARD Marc M., The Weakness of Civil Society in PostCommunist Europe, Cambridge, Cambridge University Press, 2003.

2. TODOROVA Maria, L'imaginaire des Balkans, Paris, Éditions de l'EHESS, 2011.

3. Ce dernier était d'ailleurs déjà publié à l'identique sous forme d'un dossier dans Europe-Asia Studies, vol. 69, $\mathrm{n}^{\circ}$ 9, 2017.

4. KRAMER Mark, «The International Context of Mass Political Unrest in the Balkans - Conceptual Issues and Perspectives ", dans Florian Bieber, Dario Brentin (dir.), Social Movements in the Balkans: Rebellion and Protest from Maribor to Taksim, Londres, Routledge, 2018, p. 158-187.

5. BIEBER Florian, BRENTIN Dario, «Social Movements and Protests in Southeast Europe. A new tragedy of the commons? ", dans Bieber, Brentin (dir.), Social Movements in the Balkans, op. cit., p. 1. 
6. FAGAN Adam, SIRCAR Indraneel, «Introduction: Activist Citizenship in Southeast Europe », dans Adam Fagan, Indraneel Sircar (dir.), Activist Citizenship in Southeast Europe, Londres, Routledge, 2018, p. 2.

7. HARDIN Garret, « The Tragedy of the Commons », Science, vol. 162, n 3859, 1968, p. 1243-1248.

8. BIEBER, BRENTIN, « Social Movements and Protests in Southeast Europe », art. cité, p. 8.

9. STUBBS Paul, « Networks, Organisations, Movements: Narratives and Shapes of Three Waves of Activism in Croatia ", Polemos: časopis za interdisciplinarna istraživanja rata i mira, vol. XV, $\mathrm{n}^{\circ} 30$, 2012, p. 11-32.

10. JACOBSSON Kerstin, SAXONBERG Steven (dir.), Beyond NGO-ization: The Developement of Social Movements in Central and Eastern Europe, Londres, Routledge, 2013.

11. BIEBER, BRENTIN, «Social Movements and Protests in Southeast Europe », art. cité, p. 1.

12. TILly Charles, TARRow Sidney, Contentious Politics: Second Edition, Revised and Updated. Oxford, Oxford University Press, 2015.

13. GUEORGUIEVA Valentina, "The 'Stronger State' and Counter-Democracy. Bulgarian Street Protests, 2012-2013, in the Accounts of Participants ", dans Bieber, Brentin (dir.), Social Movements in the Balkans, op. cit., p. 57.

14. WIMMEN Heiko, "Divided They Stand: Peace Building, State Reconstruction and Informal Political Movements in Bosnia-Herzegovina, 2005-2013 », dans Bieber, Brentin (dir.), Social Movements in the Balkans, op. cit., p. 9.

15. BIEBER, BRENTIN, « Social Movements and Protests in Southeast Europe », art. cité, p. 4.

16. ISIN Engin F., «Citizenship in Flux: The Figure of the Activist Citizen », Subjectivity, vol. 29, 2009, p. 367-388 (384).

17. LEFEBVRE Henri, La production de l'espace, Paris, Anthropos, 1974.

18. GoLDSTEIN Piotr, «Post-Yugoslav Everyday Activism(s). A Different Form of Activist Citizenship », dans Fagan, Sircar (dir.), Activist Citizenship in Southeast Europe, op. cit., p. 119.

19. TILly Charles, La France conteste de 1600 à nos jours, Paris, Fayard, 1986.

20. BERK Ksenija, «At the Crossroads of Cultural and Ideological Exchange. Behind the Visual Communications of 2012/2013 Slovene Protests ", dans Bieber, Brentin (dir.), Social Movements in the Balkans, op. cit., p. 79-95.

21. REINPRECHT Astrid, «Between Europe and the Past. Collective Identification and Diffusion of Student Contention to and from Serbia », dans Fagan, Sircar (dir.), Activist Citizenship in Southeast Europe, op. cit., p. 26-46.

22. MILAN Chiara, «Reshaping Citizenship through Collective Action: Performative and Prefigurative Practices in the 2013-2014 Cycle of Contention in Bosnia \& Hercegovina ", dans Fagan, Sircar (dir.), Activist Citizenship in Southeast Europe, op. cit., p. 10-25.

23. KIRN Gal, «Maribor's Social Uprising in the European Crisis: from Antipolitics of People to Politicisation of Periphery's Surplus Population », dans Bieber, Brentin (dir.), Social Movements in the Balkans, op. cit., p. 30-47.

24. TOPLIŠEK Alen, tHOMASSEN Lasse, «From Protest to Party: Horizontality and Verticality on the Slovenian Left », dans Fagan, Sircar (dir.), Activist Citizenship in Southeast Europe, op. cit., p. 47-64.

25. milan Chiara, oikonomaKis Leonidas, « Not Seeing the Forest for the Trees. From Single-Issue Protest to Resonant Mass-Movements in Greece, Turkey and Bosnia-Herzegovina ", dans Bieber, Brentin (dir.), Social Movements in the Balkans, op. cit., p. 113.

26. DOLENEC Danijela, DoolAn Karin, TOMAŠEvić Tomislav, «Contesting Neoliberal Urbanism on the European Semi-periphery: The Right to the City Movement in Croatia », dans Fagan, Sircar (dir.), Activist Citizenship in Southeast Europe, op. cit., p. 65-93.

27. PLEVRIS Kostis, "The Spaces of Social Mobilisation in Greece», dans Bieber, Brentin (dir.), Social Movements in the Balkans, op. cit., p. 66-78. 
28. LEKIĆ-SUBAŠı́́ Željka, "Social Media and 'Balkans' Spring », dans Bieber, Brentin (dir.), Social Movements in the Balkans, op. cit., p. 108.

29. TATAR Marius I., "Are the Balkans Different? Mapping Protest Politics in Post-Communist Southeastern Europe", dans Bieber, Brentin (dir.), Social Movements in the Balkans, op. cit., p. 131-157.

30. GUEORGUIEVA, « Bulgarian Street Protests », art. cité, p. 50.

31. BAĆA Bojan, «'We Are All Beranselo': Political Subjectivation as an Unintended Consequence of Activist Citizenship », dans Fagan, Sircar (dir.), Activist Citizenship in Southeast Europe, op. cit., p. 94-118.

32. ANA Alexandra, "The Role of the Feminist Movement Participation during the Winter 2012 Mobilisations in Romania », dans Fagan, Sircar (dir.), Activist Citizenship in Southeast Europe, op. cit., p. 156.

\section{INDEX}

Index géographique : Balkans

\section{AUTEURS}

\section{FABIO GIOMI}

CNRS, CETOBaC

fabio.giomi[at]gmail.com

JULIA NIETSCH

EHESS, CETOBaC

julia.nietsch[at]ehess.fr 